\title{
11. The Procedure Division
}

The program instructions are written in the Procedure Division - organised in a similar way to a book or a report written in English.

The Procedure Division may be divided into Sections - each with a heading; these are approximately equivalent to Chapters in a book. Just as you would not generally have chapters in a short essay - a short program will not usually have sections.

The program is further divided into paragraphs; these would correspond to the paragraphs in an essay or report.

Each paragraph must be given a suitable heading (see lesson 7 for permissible names).

Always divide your program into paragraphs (if you are using sections - then these should be split into paragraphs).

A paragraph is made up of sentences - each one finishing with a full-stop.

A sentence consists of one or more program statements - each one an instruction to carry out a single task.

A statement consists of one or more $\mathrm{COBOL}$ command words (sometimes called Verbs) - e.g. - DISPLAY, COMPUTE, ACCEPT - together with additional information, such as datanames.

\section{Exercise}

1. Give some examples of permitted paragraph names (see lesson 7).

2. Give 4 examples of:
a) statements;
b) sentences;
c) paragraphs.

3. Give one advantage of splitting a program into headed Sections or Paragraphs.

4. Give three examples of $\mathrm{COBOL}$ statements (look at the example programs on lessons 5 and 6).

5. Place the following in the correct order (from large to small): statement, section, division, paragraph, sentence. 